\title{
Backstepping Design for Active Control of Surge in a Centrifugal Compressor Supported by Magnetic Bearings and in the Presence of Piping Acoustics
}

\footnotetext{
A Thesis

Presented to

the Faculty of the School of Engineering and Applied Science

University of Virginia

In Partial Fulfillment

of the requirements for the Degree

Master of Science (Electrical Engineering)

by

Clive Ntuli

May 2013
} 
(C) 2013 Clive Ntuli 


\section{Approval Sheet}

This Thesis is submitled in partial fultillment of the requirements for the degree of Master of Science (Electrical Engineering)

$\frac{\text { Clive Ntuli }}{\text { AUTHOR }}$

This Thesis has been read and approved by the Examining Committee:

Zongli Lin. Adviser

\begin{tabular}{c} 
Zongli Lin, Adviser \\
Gang Tao, Committee Chair \\
Se Young Yoon \\
\hline
\end{tabular}

Accepted for the School of Engineering and Appljed Science:

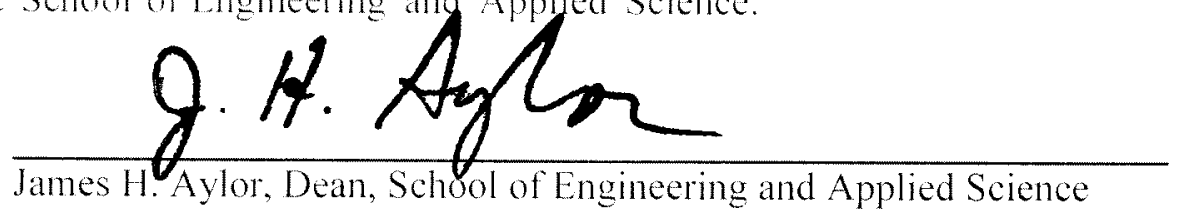

May 2013 


\section{Abstract}

As the operating point of a compressor goes below the minimum flow limit point on a compressor characteristic curve, the compressor operation transitions from a stable region to an unstable region. In the unstable region, a phenomenon called surge happens. Surge is characterized by low frequency and large amplitude oscillations in the mass flow rate and exhaust pressure rise. Because of its violent vibrations, surge can cause devastating damage to a compressor.

Active magnetic bearings (AMBs) have been utilized in recent years to provide control solutions that can actively stabilize surge. Using radial AMBs for rotor levitation and an axial AMB for modulation of the impeller tip clearance, surge can be stabilized resulting in the achievement of the highest pressure rise possible in a compressor. The control solutions provided by AMBs can also protect a compressor against damage caused by surge.

Numerous control methods using AMBs have been studied in the stabilization of surge. Some of the control methods explored include $H_{\infty}$ control, stabilization with mass flow feedback, sliding mode control and backstepping. This thesis explores the backstepping control method. Backstepping is preferred because of its ability to increase the stable operating range on the characteristic curve. In this thesis, a four state nonlinear model that was developed for a centrifugal compressor supported by AMBs is used. A surge controller is developed using backstepping design and simulation results that demonstrate promising stabilization of surge are presented. 


\section{Acknowledgments}

I would like to express my sincere appreciation to my adviser, Prof. Zongli Lin, for making this thesis a success. This thesis was a success because of the knowledge I gained from the Nonlinear Systems class which he teaches. I would also like to appreciate how he has kept on motivating me throughout. I have benefited tremendously from his knowledge and wisdom.

I would also like to thank Dr. Se Young Yoon who has always been there to answer my questions and to share his knowledge about the compressor test rig. Sometimes the simulations never made sense but with his help, debugging was always made easier. Dr. Yoon's problem solving skills were very helpful and I benefited invaluably from working alongside him.

A special thank you also goes to The Rotating Machinery and Controls Laboratory (ROMAC), for providing the compressor test rig from which the model and parameter values that were used in this thesis were experimentally determined.

Lastly, no words can describe how I am appreciative of my family for the love, encouragement and support I have always received. My family is truly a blessing. 


\section{Contents}

Contents $\quad$ iv

List of Tables . . . . . . . . . . . . . . . . . . . . v v

List of Figures . . . . . . . . . . . . . . . . . . . vi vi

1 Introduction $\quad 1$

1.1 Background ........................... 1

1.2 Objectives and Scope . . . . . . . . . . . . . . . . . . . . . . 7

1.3 Thesis Outline . . . . . . . . . . . . . . . . . . . . . . 7

2 Engineering Analysis $\quad 9$

2.1 Compressor Model . . . . . . . . . . . . . . . . . . . . 9 9

2.2 Effects of Impeller Tip Clearance . . . . . . . . . . . . . . . . . . . . 14

2.3 Linearization of Impeller Tip Clearance Effects . . . . . . . . . . . . . 17

2.4 Piping Model . . . . . . . . . . . . . . . . . . . . . . . . 19

2.5 Overall Model . . . . . . . . . . . . . . . . . . 20

3 Controller Derivation $\quad 22$

3.1 Integrator Backstepping . . . . . . . . . . . . . . . . . . . . 22

3.2 Backstepping Design for Surge Control . . . . . . . . . . . . . . 23

3.3 Simulation Results . . . . . . . . . . . . . . . . . . 33

4 Conclusions and Future Work $\quad 37$

4.1 Conclusions . . . . . . . . . . . . . . . . . . . . . 37

4.2 Future Experimental Work . . . . . . . . . . . . . . . . 38

4.3 Future Research Work . . . . . . . . . . . . . . . . . . 39

4.4 Economic Viability of Active Magnetic Bearings . . . . . . . . . . . 39

$\begin{array}{ll}\text { Bibliography } & 41\end{array}$ 


\section{List of Tables}

2.1 Model parameter values $[1] \ldots \ldots \ldots 21$ 


\section{List of Figures}

1.1 A cross section of the axial compressor $[2] \ldots \ldots \ldots \ldots$

1.2 A cross section of the centrifugal compressor $[2] \ldots \ldots$

1.3 A stainless steel impeller . . . . . . . . . . . . . . . . . . 3

1.4 A comparison of radial and mixed-flow designs [3] . . . . . . . . 4

1.5 Compressor characteristic curves with increasing speed $\mathrm{N}$ and demand load curves at the compressor exhaust $[1] \ldots \ldots \ldots \ldots$

2.1 Compressor system schematic as developed in $[4] \ldots \ldots$

2.2 Fitted characteristic curve of the compressor at 16,290 rpm [1] . . . 12

2.3 Fitted of the throttle valve constant at $16,290 \mathrm{rpm}[1] \ldots \ldots$

3.1 Demonstration of a surge interval with controller turned off . . . . . . 34

3.2 Controller engaged and surge interval stabilized . . . . . . . . 34

3.3 Demonstration of continued surge . . . . . . . . . . . . . 35

3.4 An engaged controller stabilizes surge . . . . . . . . . . 35

3.5 Demonstration of surge control on the compressor characteristic curve 36 


\section{Chapter 1}

\section{Introduction}

\section{$1.1 \quad$ Background}

Compressors find their use in applications that require the pressurization of compressible fluids, mainly gases. Such applications range from small scale use in home refrigerators to large scale use in petrochemical industries. There are basically two broad groups of compressors and the grouping is based on the compression mode [3]. The first group of compressors uses the intermittent mode of compression in which a specific quantity of gas is injected into the compressor, worked on, and then discharged. This process is repeated as necessary. Compressors that use the intermittent compression mode are called positive displacement compressors. From the gas laws, it follows that an increase in pressure can be achieved by reducing the volume of the input gas in positive displacement compressors. The second group of compressors is characterized by continuous flow, in which gas is continuously injected into the compressor, worked on, and then discharged without the flow being interrupted. This group is known as dynamic compressors. In dynamic compressors, an increase in pressure is achieved by transferring energy from a fast moving impeller to the gas. The kinetic energy of the gas is then converted into potential energy by means of a 


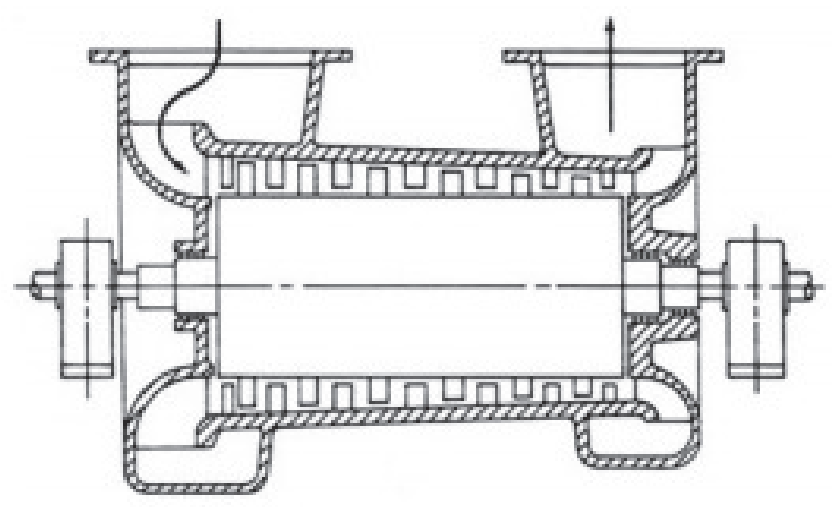

Figure 1.1: A cross section of the axial compressor [2]

diffuser. The potential energy in this case is the static pressure. Under these two groups of compressors, there are other subgroups and the nature of their operation is discussed in [3] and [5].

Dynamic compressors can be further divided into three types depending on the direction in which the gas flows out of the compressor [3]. Usually, gas enters the compressor axially, parallel to the axis of rotation of the rotor. In axial compressors the gas leaves the compressor impeller in the same manner, axially, as shown in Figure 1.1. Axial compressors are typically multi-stage; they have multiple rows of impellers. The rows of blades in axial compressors alternate between stator blade rows and rotor impeller blade rows. The stator blade rows are fixed while the rotor impeller blade rows are the ones that rotate. At the same flow rating, axial compressors are known to provide higher efficiency than other types of dynamic compressors [3].

The second type of dynamic compressors is the radial or centrifugal compressor as shown in Figure 1.2. In centrifugal compressors the gas leaves the compressor impeller radially, going into the stationary diffuser. In the diffuser, the gas is slowed down from the high velocities it would have acquired through the impeller, leading to the build up in pressure. The pressure build up in the diffuser is dependent on the backward leaning of the impeller blades. As can be seen in Figure 1.3, the impeller blades lean backwards. The more the blades lean backwards, the lower the pressure conversion 


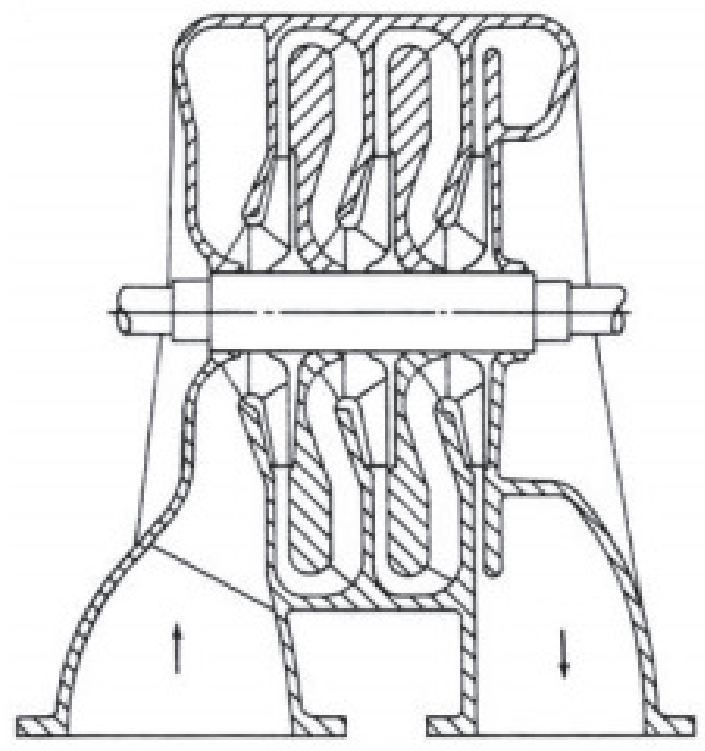

Figure 1.2: A cross section of the centrifugal compressor [2]

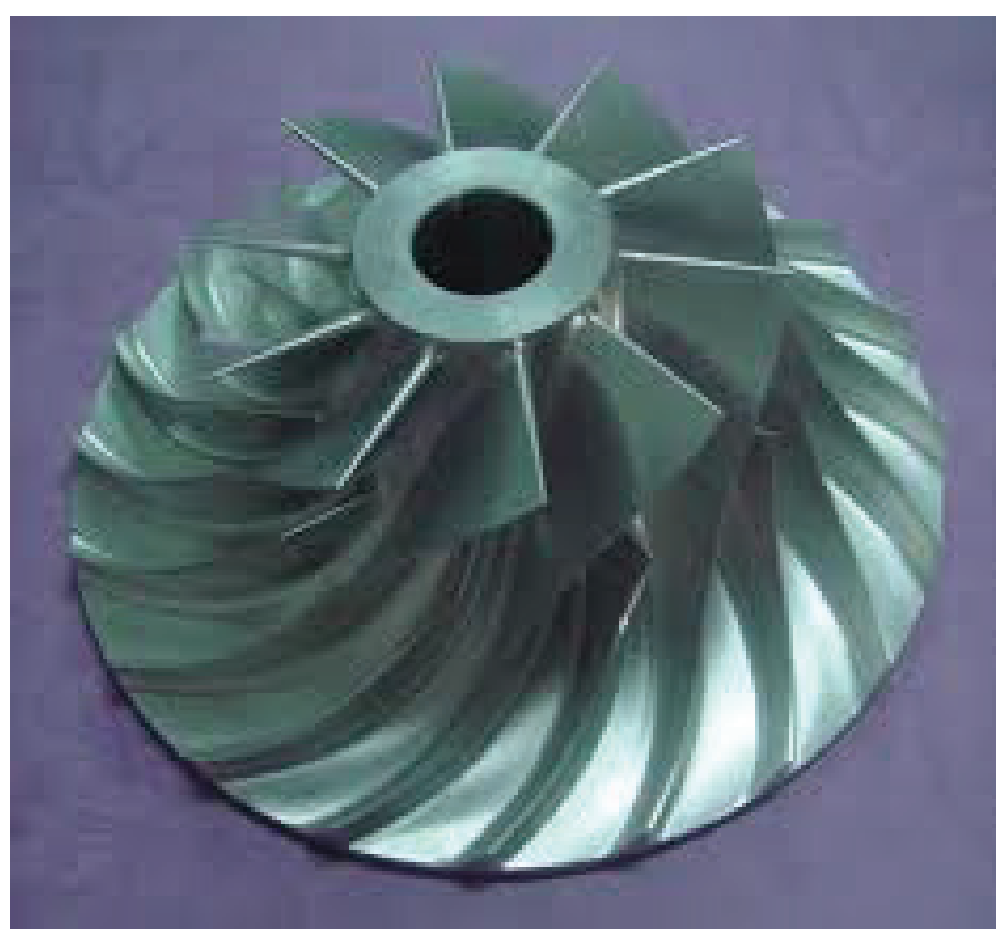

Figure 1.3: A stainless steel impeller

in the impeller but the higher the pressure conversion in the diffuser. The design of impellers is presented in [6]. 


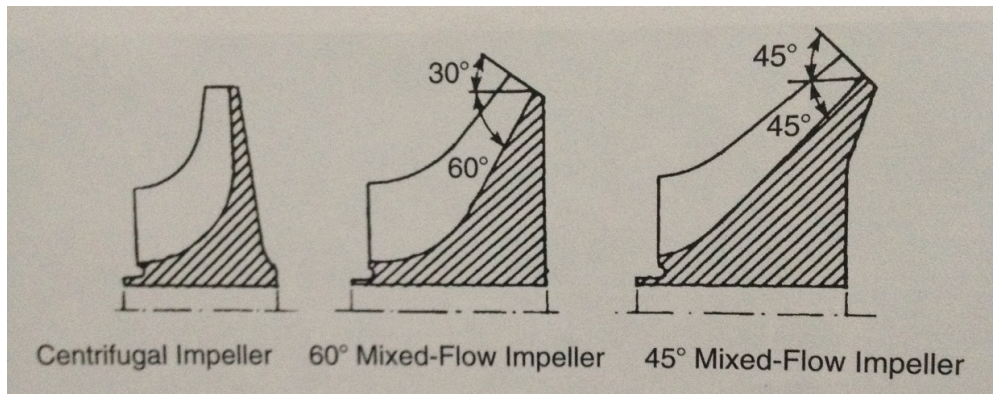

Figure 1.4: A comparison of radial and mixed-flow designs [3]

The third and last type of dynamic compressors is the rarely used mixed-flow compressor. Mixed-flow compressors are hybrids of axial flow and radial flow compressors. Whilst they achieve energy transfer exactly the same way as centrifugal compressors, mixed-flow compressors achieve higher flow rates than centrifugal compressors according to [3]. A comparison of radial-flow and mixed-flow designs is highlighted in Figure 1.4 .

As with all mechanical machines, designing compressors for optimum performance is an area of ever-growing interest in industry and academia. For compressors, the measure of performance is mainly determined from the compressor's characteristic curve. A characteristic curve is a plot of the discharge pressure rise against the inlet flow rate, for a compressor operating at steady-state and at a particular constant operating speed. Given the characteristic curve of a compressor, the operating point is found at the intersection of the characteristic curve and the demand load curve. An approach for finding the coefficients of a characteristic curve is outlined in [7]. On the characteristic curve, operating on a point that is on the left side of the minimum flow point results in a phenomenon called surge. The minimum flow point is referred to as the surge limit, because it is the lowest flow rate the compressor operation is stable. Operation of a compressor below the surge limit can result in an intense and rapid oscillating flow characterized by pressure fluctuations in the system. Surge causes violent vibrations and loud low-frequency noises in compression system. In 


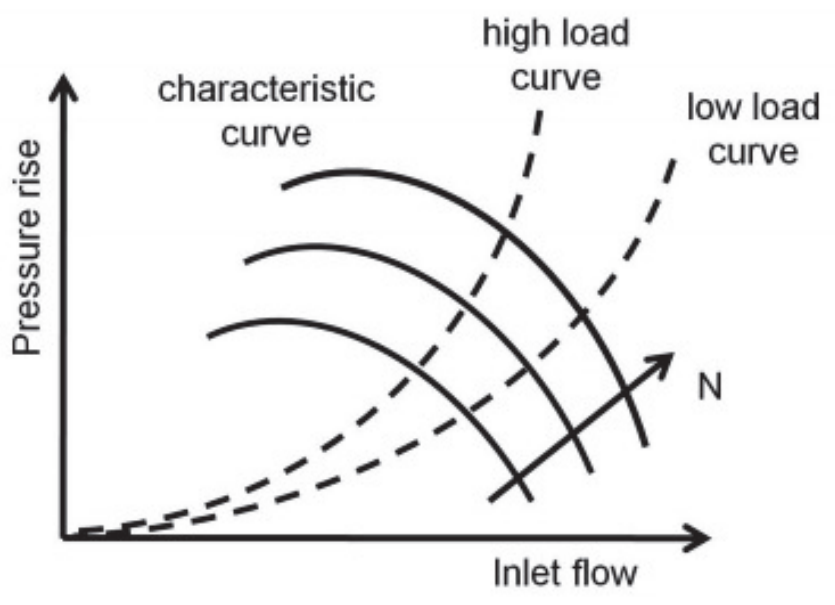

Figure 1.5: Compressor characteristic curves with increasing speed $\mathrm{N}$ and demand load curves at the compressor exhaust [1]

some machines, surge is accompanied by a squeal. Whilst most machines are designed to temporarily endure surge, prolonged and unchecked operation in surge can result in catastrophic breakdown of the machine.

Given the devastating nature of surge to a machine, numerous solutions have been developed to deal with surge. One of the obvious solutions is to avoid surge altogether. Through test-operating the compressor at various flow rates, the minimum flow rate can be identified, and with this knowledge operation can be limited to the flow range in which stable operation is guaranteed. The standard practice in industry is to place a safety margin line that is $10 \%$ away from the surge limit [8]. This approach is classified as surge avoidance. Surge avoidance can be achieved by increasing the throttle valve opening which results in an increased flow that helps maintain the stable operation of the compressor. In the event that the compressor does not have a throttle valve opening, as in process gas compressors, a recycle valve with an inter-cooler downstream of the compressor discharge can be installed in the compressor [9]. As the surge limit is approached, the recycle valve is opened to allow more exhaust flow back to the suction of the compressor. Whilst surge avoidance is simple and easy to implement, the loss of efficiency and the inability to achieve high pressure rise levels 
make the solution undesirable.

Another approach that has been developed to dealing with surge involves modifying the compressor during design or adding external devices on the compressor that can actively control surge. Some of the approaches that have been studied involve the injection of air into a compressor's diffuser to stabilize the flow and the installation of variable inlet guide vanes to the suction of a centrifugal compressor [10, 11]. Employing feedback mechanisms, many researchers have come up with control methods to suppress the surge instability. Pioneered by Epstein et al, active surge control utilizes sensors and actuators to actively provide feedback control based on sensor measurements [12]. The sensor measurements are processed by a computer which then generates a control signal that is fed to the actuators. Krstic [13] theoretically showed that the backstepping control method can be used to stabilize surge and stall in a jet engine. Using pressure and flow rate measurements, Krstic developed a controller that perturbed the throttle valve to change the bifurcation property in the stall and surge regions of the jet engine.

In an almost related manner, Sanadgol [4] proposed a new method to control surge by employing active surge control but using AMBs to modulate the impeller tip clearance. In this new method, Sanadgol used radial AMBs to enable rotor levitation and an axial AMB to change the position of the rotor. The use of AMBs enabled the modulation of the impeller tip clearance even if the compressor is in operation. The modulation of the impeller can increase or reduce the pressure build up in the impeller region and this ability can make the active control of surge achievable. Detailed description of the design, manufacturing and operation of AMBs is found in [14]. A centrifugal compressor was built to perform experimental work to validate the theory Sanadgol developed [15]. The Rotating Machinery and Controls Laboratory (ROMAC) and its industrial partners sponsored the project to build the compressor.

The experimental results to validate the surge control method as proposed by 
Sanadgol was presented by Yoon in [1] and Yoon et. al in [7]. During the experimental testing, it was observed that both the closed-loop dynamics of the AMBs and the acoustic resonance of the compressor piping degraded the effectiveness of the surge controller when compared to predictions in [4]. In response to this observation, the authors in [7] and [1] incorporated the transmission line dynamics of the plenum piping acoustics. From the enhanced surge model, an $H_{\infty}$ controller was developed in which the closed-loop dynamics of the AMBs were treated as frequency dependent unstructured uncertainties. The derived controller was implemented and tested successfully in the experimental centrifugal compressor. It was demonstrated during experimental testing in $[1,7]$ that the derived controller is capable of shifting the flow rate at the surge line by as much as $23 \%$.

\subsection{Objectives and Scope}

The objective and scope of study of this thesis is to develop a controller using the backstepping control method to improve on the modulation of the impeller and increase the range of stable operation on the characteristic curve. The thesis also aims to establish the platform for experimentally evaluating the effectiveness of the developed controller.

\subsection{Thesis Outline}

This thesis consists of four chapters. The first chapter provides an introduction to compressors, surge and various approaches to control it. Chapter 1 also includes a discussion of the previous work this thesis will build on. Chapter 2 shows how the compressor model and the piping model were developed and assembled. This chapter also provides information on the compressor test rig the model detailed in this thesis is based upon. Chapter 3 discusses the controller derivation and simulation results. 
The last chapter, Chapter 4, provides a closing conclusion, recommendations for the practical implementation of the controller, possible future work and a discussion on the economic viability of AMBs. 


\section{Chapter 2}

\section{Engineering Analysis}

\subsection{Compressor Model}

The development of the compressor model that is used in this study is presented in $[4,1,7]$. This chapter shows how the model was developed starting from the famous Greitzer model. Greitzer [16] famously developed a lumped parameter model for a compression system that consisted of a compressor, a plenum and a throttle valve. The original model had four states and could be formulated as,

$$
\begin{aligned}
\frac{d \Phi_{c}}{d \tau} & =B\left(\Psi_{c}-\Psi_{p}\right), \\
\frac{d \Phi_{t h}}{d \tau} & =\frac{B}{\mathbf{G}}\left(\Psi_{p}-\Psi_{t h}\right), \\
\frac{d \Psi_{p}}{d \tau} & =\frac{1}{B}\left(\Phi_{c}-\Phi_{t h}\right), \\
\frac{d \Psi_{c}}{d \tau} & =\frac{1}{\tau}\left(\Psi_{c, s s}-\Psi_{c}\right),
\end{aligned}
$$




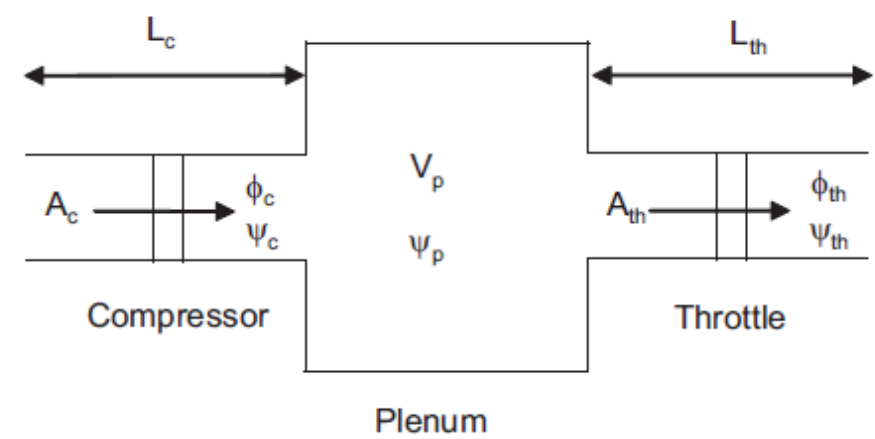

Figure 2.1: Compressor system schematic as developed in [4]

where the parameters included in Equation 2.1 are defined as,

$$
\begin{aligned}
\tau & =t \omega_{H}, \\
\omega_{H} & =\mathbf{a}_{o 1} \sqrt{\frac{A_{c}}{V_{p} L_{c}}}, \\
B & =\frac{U}{2 \omega_{H} L_{c}}, \\
\mathbf{G} & =\frac{L_{t h} A_{c}}{A_{t h} L_{c}},
\end{aligned}
$$

and $t$ is time, $\mathbf{a}_{o 1}$ the ambient speed of sound, $A_{c}$ the area of the impeller eye, $V_{p}$ the volume of the plenum, $U$ the impeller tip velocity, $A_{t h}$ the throttle valve cross sectional area, $L_{t h}$ length of throttle duct and $L_{c}$ length of the compressor duct. Figure 2.1 is a schematic of the compressor and illustrates the location of some of the parameters introduced on the right side Equation 2.2. As shown in Figure 2.1, $\Phi_{c}$ is the compressor mass flow rate, $\Phi_{t h}$ is the throttle mass flow rate, $\Psi_{p}$ is the plenum pressure rise, $\Psi_{t h}$ is the throttle pressure rise and $\Psi_{c}$ is the compressor pressure rise, all these are non-dimensional. The parameter $B$ in Equation 2.2 is a constant known as the Greitzer stability parameter and it governs the intensity of surge instability in the Greitzer model. Parameter $\omega_{H}$ is also a constant called the Helmholtz frequency. The parameter $\mathbf{G}$ measures the inertia effects in the throttle duct compared to the 
inertia effects in the compressor duct. In the Greitzer model in Equation 2.1, the non-dimensionalization of the pressure rise $\Psi$ and the mass flow rate $\Phi$ are defined as,

$$
\begin{aligned}
\Psi & =\frac{p-p_{o 1}}{\frac{1}{2} \rho_{o 1} U^{2}}, \\
\Phi & =\frac{m}{\rho_{o 1} U A_{c}} .
\end{aligned}
$$

The parameters used for non-dimensionalizing above are the absolute pressure $p$, the inlet absolute pressure $p_{o 1}$, the density of air at the inlet $\rho_{o 1}$, the impeller tip velocity $U$, the cross section area of the compressor duct $A_{c}$ and the mass flow rate $m$.

In the Greitzer model, the first dynamics in Equation 2.1a are for the conservation of momentum in the compressor duct. The dynamics for the conservation of momentum in the throttle duct are modeled by Equation 2.1b. Equation 2.1c models the dynamics for the conservation of mass in the plenum. The last equation, Equation 2.1d models rotating stall.

The Greitzer model can be simplified be neglecting the effect of $\mathbf{G}$ since the length of the throttle duct $L_{t h}$ is significantly shorter than the length of the compressor duct $L_{c}$. Also, since the performance of a centrifugal compressor is not affected by rotating stall, Equation 2.1d can be ignored completely. With these simplifications, the Greitzer model becomes,

$$
\begin{aligned}
& \dot{\Phi}_{c}=B \omega_{H}\left(\Psi_{c}-\Psi_{p}\right), \\
& \dot{\Psi}_{p}=\frac{\omega_{H}}{B}\left(\Phi_{c}-\Phi_{t h}\right) .
\end{aligned}
$$

Whilst the Greitzer model was originally developed for low pressure axial compressors, the simplified model can be successfully applied to some centrifugal compressors. The Greitzer model is renowned for its ability to capture the nonlinear surge instability in compression systems. However, Yoon [1] experimentally validated that the piping 


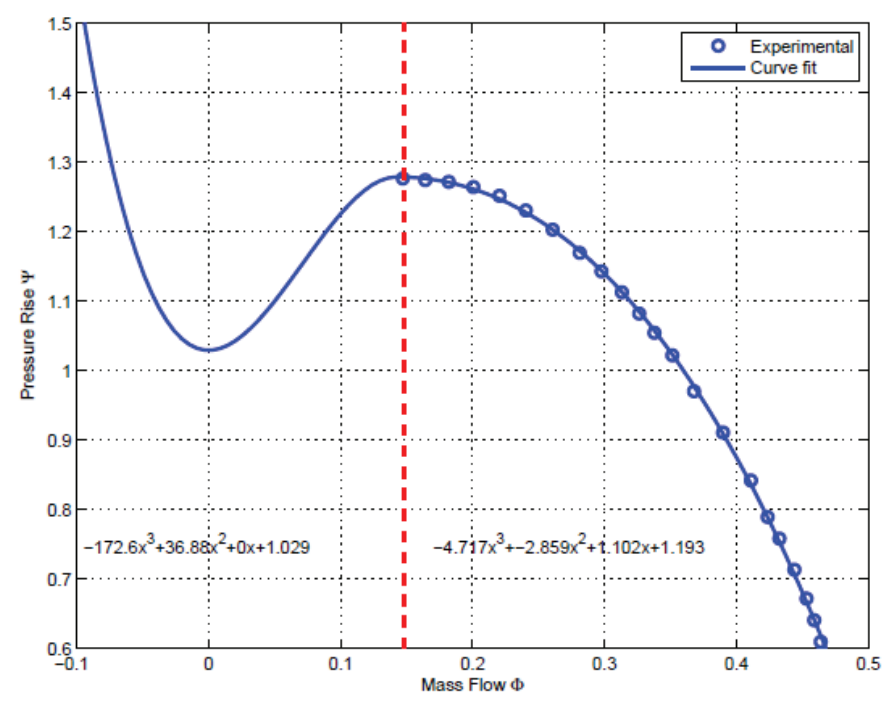

Figure 2.2: Fitted characteristic curve of the compressor at 16,290 rpm [1]

acoustics of the compression system of the centrifugal compressor generated acoustic resonances which the Greitzer model missed.

The compressor system model in Equation 2.4 assumes steady-state characteristics of the compressor flow that are given by,

$$
\Psi_{c, s s}\left(\Phi_{c}\right)=A_{1} \Phi_{c}^{3}+B_{1} \Phi_{c}^{2}+D_{1}
$$

The method to obtain the coefficients of the steady-state characteristic compressor flow in Equation 2.5 is outlined in [1]. It is important to note that this curve corresponds to the unstable region of the characteristic curve that lies on the left side of the surge limit line. The unstable region is used because ideally the controller's task is to stabilize flow in that region, so it makes sense for the controller to have information of the region in which stabilization is sought. Figure 2.2 illustrates the fitted characteristic curve at 16,290 rpm as determined by Yoon. Another important relationship in the development of the compressor model is how the throttle mass flow rate is a function of the percentage of throttle valve opening and the plenum pressure rise under subsonic 


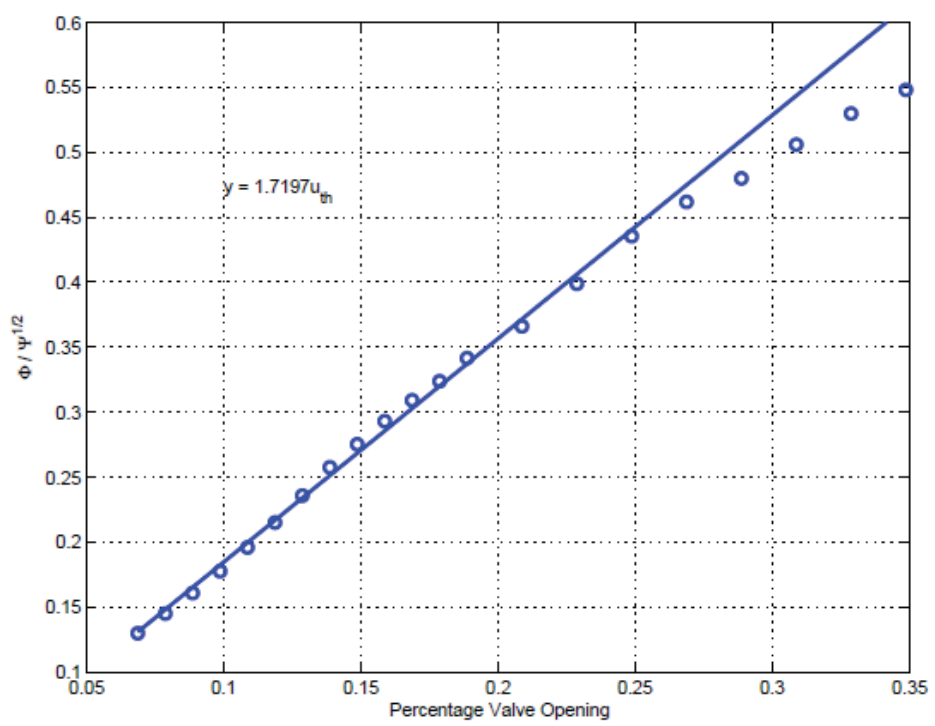

Figure 2.3: Fitted of the throttle valve constant at 16,290 rpm [1]

flow conditions [17],

$$
\Phi_{t h}=u_{t h} c_{t h} \sqrt{\Psi_{p}}
$$

The percentage throttle valve opening is $u_{t h}$ and $c_{t h}$ is the throttle valve constant that is determined experimentally. Yoon recorded steady-state pressure rises and the accompanying mass flow rates at different throttle valve openings when the compressor was operating at 16,290 rpm. Figure 2.3 shows how the fitted curve was obtained using experimental data. The Greitzer model in Equation 2.4 was applied to the experimental test rig under the following assumptions [4]:

- The compression system operates at low inlet Mach numbers.

- The pressure rises achieved by the compression system are low compared to the ambient pressure.

- Flow in the duct section is taken to be one-dimensional and incompressible. 
- The compression process in the plenum section is isentropic and with uniform pressure distribution.

- Fluid velocities in the plenum are considered to be negligible.

- Plenum dimensions are much smaller than the wavelength of the acoustic waves related to surge.

\subsection{Effects of Impeller Tip Clearance}

The tip clearance modulation was achieved by a thrust AMB which guaranteed very high precision and enough bandwidth. Different studies [18, 19, 20] have shown different effects in the compressor flow to variations in the impeller tip clearance. With knowledge that these differences were largely as a result of the difficulty in measuring and controlling tip clearances in compressors that do not use AMBs, Sanadgol [4] formulated the mathematical derivations that described the effects of impeller tip clearance modulation on the compressor model. Yoon [1] provided some corrections to the derivations through experimentation. It was observed that for a $1 \%$ change in the compressor efficiency $\Delta \eta$ there is a variation of $1.43 \%$ in the ratio between axial impeller tip clearance $c l$ and the blade height at the impeller exit $b_{2}$. This relationship can be expressed as,

$$
\frac{\Delta \eta}{\eta} \approx \frac{0.7 c l}{b_{2}}
$$

Solving for efficiency $\eta$ in Equation 2.7 as a function of the impeller tip clearance, impeller blade height and efficiency at zero clearance $\eta_{0}$, we get

$$
\eta=\frac{\eta_{0}}{1+\frac{0.7 c l_{n}}{b_{2}}}
$$


Since the efficiency at zero impeller tip clearance is impossible to obtain because of physical limitations, a turnaround of modifying Equation 2.8 to measure efficiency in terms of nominal efficiency can help in evaluating the equation. The nominal efficiency $\eta_{n}$ at the nominal tip clearance $c l_{n}$, the design value for the compressor, can then be expressed as,

$$
\eta_{n}=\frac{\eta_{0}}{1+\frac{0.7 c l_{n}}{b_{2}}} .
$$

Solving for $\eta_{0}$ in Equation 2.9 and substituting it in Equation 2.8, we get the expression for the compressor efficiency as,

$$
\eta=\frac{\eta_{n}\left(1+\frac{0.7 c l_{n}}{b_{2}}\right)}{1+\frac{0.7 c l}{b_{2}}}
$$

Simplifying the expression in Equation 2.10 by introducing two new variables we get,

$$
\eta=\frac{\eta_{n}}{1-k_{0} \frac{\delta_{c l}}{b_{2}}},
$$

where the two introduced variables are defined as,

$$
\begin{aligned}
& \delta_{c l}=c l_{n}-c l \\
& k_{0}=\frac{0.7}{1+\frac{0.7 c l_{n}}{b_{2}}} .
\end{aligned}
$$

The variable $\delta_{c l}$ is clearly the variation of the impeller tip clearance from the nominal position $c l_{n}$ whilst $k_{0}$ becomes an impeller tip clearance coefficient. 
With reference to perfect gas and isentropic compression assumptions, the totalto-static isentropic efficiency of the compressor is expressed as,

$$
\eta=\frac{T_{o 1} C_{p}\left(\left(\frac{p_{c}}{p_{o 1}}\right)^{\frac{\gamma-1}{\gamma}}-1\right)}{\Delta h_{o c, \text { ideal }}}
$$

where $T_{o 1}$ is the inlet stagnation temperature, $C_{p}$ is the specific heat at constant pressure, $p_{o 1}$ is the ambient pressure, $p_{c}$ the compressor output pressure at the compressor exhaust, $\Delta h_{o c, \text { ideal }}$ is the total specific enthalpy delivered to the fluid and $\gamma$ is the specific heat ratio. At the nominal tip clearance the nominal total-to-static isentropic efficiency can be consistently expressed as,

$$
\eta_{n}=\frac{T_{o 1} C_{p}\left(\left(\frac{p_{c, s s}}{p_{o 1}}\right)^{\frac{\gamma-1}{\gamma}}-1\right)}{\Delta h_{o c, \text { ideal }}},
$$

where $p_{c, s s}$ is the compressor output pressure at nominal clearance.

Since the compressor exhaust pressure ratio $\psi_{c}$ and the compressor exhaust pressure ratio at nominal tip clearance $\psi_{c, s s}$ are defined as,

$$
\begin{aligned}
\psi_{c} & =\frac{p_{c}}{p_{o 1}}, \\
\psi_{c, s s} & =\frac{p_{c, s s}}{p_{o 1}},
\end{aligned}
$$

a new expression of the compressor exhaust pressure ratio $\psi_{c}$ that is as a result of substituting $\eta$ and $\eta_{n}$ in Equation 2.13 and Equation 2.15 respectively, into Equation 2.11, and solving for the compressor exhaust pressure ratio $\psi_{c}$ yields,

$$
\psi_{c}=\left(1+\frac{\psi_{c, s s}^{\frac{\gamma-1}{\gamma}}-1}{1-k_{0} \frac{\delta_{c l}}{b_{2}}}\right)^{\frac{\gamma}{\gamma-1}} .
$$


The nominal compressor exhaust pressure ratio $\psi_{c, s s}$ can be obtained from the characteristic curve of the compressor at the specified compressor flow rate. Using Equation 2.3 , the non-dimensional compressor pressure rise $\Psi_{c}$ can be expressed as a function of the nominal compressor exhaust pressure ratio $\psi_{c, s s}$ and the variation in the impeller tip clearance $\delta_{c l}$ defined in Equation 2.12. The expression is,

$$
\Psi_{c}=\frac{p_{o 1}}{\frac{1}{2} \rho_{o 1} U^{2}}\left(\left(1+\frac{\left(\frac{\frac{1}{2} \rho_{o 1} U^{2}}{p_{o 1}} \Psi_{c, s s}+1\right)^{\frac{\gamma-1}{\gamma}}-1}{1-k_{0} \frac{\delta_{c l}}{b_{2}}}\right)^{\frac{\gamma}{\gamma-1}}-1\right)
$$

The density of air at the inlet $\rho_{o 1}$ and the impeller tip velocity $U$ have been introduced already in Equation 2.3 during non-dimensionalizing. The compressor pressure rise in Equation 2.17 is substituted into the simplified Greitzer model in Equation 2.4 and that concludes the inclusion of impeller tip clearance modulation in the compressor model.

\subsection{Linearization of Impeller Tip Clearance Effects}

The relationship between the non-dimensional compressor pressure rise and the variation in the impeller tip clearance derived in Equation 2.16 is highly nonlinear and makes it inconvenient for deriving a surge controller. The linearization of the impeller tip clearance effects about the nominal tip clearance where $\delta_{c l}$ is zero can help simplify surge controller derivation. Defining the right hand side of Equation 2.16 as $f\left(\delta_{c l}\right)$ and then using the Taylor expansion theorem, Equation 2.16 can be written as,

$$
\psi_{c}=\left.f\left(\delta_{c l}\right)\right|_{\delta_{c l}=0}+\left.\frac{\partial f\left(\delta_{c l}\right)}{\partial \delta_{c l}}\right|_{\delta_{c l}=0} \delta_{c l}+\left.\frac{1}{2 !} \frac{\partial^{2} f\left(\delta_{c l}\right)}{\partial \delta_{c l}^{2}}\right|_{\delta_{c l}=0} \delta_{c l}^{2}+\ldots
$$

and throwing away the high order terms, the relationship between the compressor pressure rise and the impeller tip clearance becomes linear. The resulting expression 
of the linear approximation becomes,

$$
\hat{\psi}_{c}=\psi_{c, s s}-\frac{\gamma}{\gamma-1} \frac{k_{0}}{b_{2}} \psi_{c, s s}^{\frac{1}{\gamma}}\left(\psi_{c, s s}^{\frac{\gamma-1}{\gamma}}-1\right) \delta_{c l}
$$

Equation 2.19 above can be further simplified by defining a new variable $k_{c l}$ as,

$$
k_{c l}=-\frac{\gamma}{\gamma-1} \frac{k_{0}}{b_{2}} \psi_{c, s s}^{\frac{1}{\gamma}}\left(\psi_{c, s s}^{\frac{\gamma-1}{\gamma}}-1\right)
$$

resulting in a more cleaner and simpler linear approximation of the compressor pressure rise, given as

$$
\hat{\psi}_{c}=\psi_{c, s s}+k_{c l} \delta_{c l}
$$

Non-dimensionalizing the compressor pressure rise the same way done in Equation 2.3 we get,

$$
\hat{\Psi}_{c}=\frac{p_{o 1}\left(\hat{\psi}_{c}-1\right)}{\frac{1}{2} \rho_{o 1} U^{2}} .
$$

Substituting the linear approximation of the compressor pressure rise in Equation 2.21 into Equation 2.22 and non-dimensionalizing the steady state compressor pressure rise $\psi_{c, s s}$, we arrive at

$$
\hat{\Psi}_{c}=\Psi_{c, s s}+\frac{p_{o 1}}{\frac{1}{2} \rho_{o 1} U^{2}} k_{c l} \delta_{c l}
$$

If the non-dimensional compressor pressure rise $\Psi_{c}$ in the simplified Greitzer model in Equation 2.4 is substituted with the approximation in Equation 2.23 that includes 
linear effects of modulating the impeller we get the final compressor model given by,

$$
\begin{aligned}
& \dot{\Phi}_{c}=B \omega_{H}\left(\Psi_{c, s s}+\frac{p_{o 1}}{\frac{1}{2} \rho_{o 1} U^{2}} k_{c l} \delta_{c l}-\Psi_{p}\right), \\
& \dot{\Psi}_{p}=\frac{\omega_{H}}{B}\left(\Phi_{c}-\Phi_{t h}\right) .
\end{aligned}
$$

\subsection{Piping Model}

The model in Equation 2.24 is not yet final and it has to include the piping model that is part of the compressor system according to experimental findings by Yoon [1]. Referencing the transmission line model that was studied in [21] the dynamic piping equations can be written in single mode state space representation as,

$$
\left[\begin{array}{c}
\dot{p}_{t h} \\
\dot{q}_{p}
\end{array}\right]=\left[\begin{array}{cc}
0 & \frac{-Z \pi}{2 d} \\
\frac{\pi}{2 d Z} & 8
\end{array}\right]\left[\begin{array}{c}
p_{t h} \\
q_{p}
\end{array}\right]+\left[\begin{array}{cc}
0 & \frac{-2 Z}{d} \\
\frac{2}{Z d} & 0
\end{array}\right] \mathbf{G}\left[\begin{array}{c}
p_{p} \\
q_{t h}
\end{array}\right],
$$

where $p_{p}$ and $q_{p}$ are the upstream pressure and volumetric flow rate, respectively, and $p_{t h}$ and $q_{t h}$ are the downstream pressure and volumetric flow rate. The remaining parameters are $Z$, the inviscid impedance constant and $d$, the line dissipation number. After adding a steady-state correction to the model that is in [7], the pipeline dynamics can be represented in state space as,

$$
\left[\begin{array}{c}
\dot{p}_{t h} \\
\dot{q}_{p}
\end{array}\right]=\left[\begin{array}{cc}
0 & A_{12} \\
A_{21} & A_{22}
\end{array}\right]\left[\begin{array}{c}
p_{t h} \\
q_{p}
\end{array}\right]+\left[\begin{array}{cc}
0 & B_{12} \\
B_{21} & B_{22}
\end{array}\right] \mathbf{G}\left[\begin{array}{c}
p_{p} \\
q_{t h}
\end{array}\right] .
$$

The definitions of the $Z$ and $d$ parameters in Equation 2.25, and the detailed derivation of the coefficients $A_{i j}$ and $B_{i j}$ in Equation 2.26 can be found in [7].

Applying the assumption that the change in the density of the gas due to fluctuations in pressure and temperature is negligibly small, Equation 2.26 can be non- 
dimensionalized using Equation 2.3 to give,

$$
\left[\begin{array}{l}
\dot{\Psi}_{t h} \\
\dot{\Phi}_{p}
\end{array}\right]=\left[\begin{array}{cc}
0 & \frac{2 A_{12} A_{c}}{\rho_{u} U} \\
\frac{A_{21} \rho_{u} U}{2 A_{c}} & A_{22}
\end{array}\right]\left[\begin{array}{l}
\Psi_{t h} \\
\Phi_{p}
\end{array}\right]+\left[\begin{array}{cc}
0 & \frac{2 B_{12} A_{c}}{\rho_{u} U} \\
\frac{B_{21} \rho_{u} U}{2 A_{c}} & B_{22}
\end{array}\right]\left[\begin{array}{c}
\Psi_{p} \\
\Phi_{t h}
\end{array}\right]+\left[\begin{array}{c}
0 \\
\frac{\rho_{u} p_{o 1}}{\rho_{o 1} U A_{c}}\left(A_{21}+B_{21}\right)
\end{array}\right] .
$$

\subsection{Overall Model}

Using the relationship between the pressure and mass flow rate in the throttle valve section given by Equation 2.6 and the steady-state characteristics given by Equation 2.5, the piping model in Equation 2.27 can be combined with the Greitzer model in Equation 2.4 to give the overall model in Equation 2.28,

$$
\begin{aligned}
\dot{\Phi}_{c}= & B \omega_{H}\left(A_{1} \Phi_{c}^{3}+B_{1} \Phi_{c}^{2}+D_{1}+\frac{p_{o 1}}{\frac{1}{2} \rho_{o 1} U^{2}} k_{c l} \delta_{c l}-\Psi_{p}\right), \\
\dot{\Psi}_{p}= & \frac{\omega_{H}}{B}\left(\Phi_{c}-\Phi_{p}\right) \\
\dot{\Psi}_{t h}= & \frac{2 A_{12} A_{c}}{\rho_{u} U} \Phi_{p}+\frac{2 B_{12} A_{c}}{\rho_{u} U} u_{t h} c_{t h} \sqrt{\Psi_{t h}}, \\
\dot{\Phi}_{p}= & \frac{A_{21} \rho_{u} U}{2 A_{c}} \Psi_{t h}+A_{22} \Phi_{p}+\frac{B_{21} \rho_{u} U}{2 A_{c}} \Psi_{p}+\frac{\rho_{u} p_{o 1}}{\rho_{o 1} U A_{c}}\left(A_{21}+B_{21}\right)+\ldots \\
& +B_{22} u_{t h} c_{t h} \sqrt{\Psi_{t h}} .
\end{aligned}
$$

The overall model is a nonlinear system that has the state variables: compressor mass flow rate, plenum pressure rise, throttle section pressure rise and plenum mass flow rate. Yoon [1] experimentally validated the model in Equation 2.28 using the parameter values given in Table 2.1. At 16,290 rpm and 21\% throttle valve opening, Yoon observed a good agreement between the model and the experimental test rig, in both magnitude and phase responses. 
Table 2.1: Model parameter values [1]

\begin{tabular}{cccc}
\hline \hline Parameter & Symbol & Unit & Parameter Value \\
\hline Comp. duct length & $L_{c}$ & $\mathrm{~m}$ & 1.86 \\
Comp. duct cross. area & $A_{c}$ & $\mathrm{~m}^{2}$ & 0.0082 \\
Corrected $A_{1}$ coeff. & $A_{1}$ & - & -172.6 \\
Corrected $B_{1}$ coeff. & $B_{1}$ & - & 36.88 \\
Corrected $D_{1}$ coeff. & $D_{1}$ & - & 1.029 \\
Design tip clearance & $c_{n}$ & $\mathrm{~mm}$ & 0.6 \\
Greitzer stab. parameter & $B$ & - & 0.44 \\
Helmhotz freq. & $\omega_{H}$ & $\mathrm{rad} / \mathrm{s}$ & 80.1 \\
Impeller tip speed & $U$ & $\mathrm{~m} / \mathrm{s}$ & 213.24 \\
Impeller blade height & $b_{2}$ & $\mathrm{~mm}$ & 8.21 \\
Inlet pressure & $p_{o 1}$ & $\mathrm{~Pa}$ & 101.325 \\
Inlet gas density & $\rho_{o 1}$ & $\mathrm{Kg} / \mathrm{m}^{3}$ & 1.165 \\
Line dissipation number & $d$ & - & $2.38 \times 10^{-5}$ \\
Line impedance constant & $Z$ & $\mathrm{~Pa} \mathrm{~s} / \mathrm{m}$ & $4.39 \times 10^{4}$ \\
Plenum volume & $V_{p}$ & $\mathrm{~m}^{3}$ & 0.049 \\
Pipeline length & $L$ & $\mathrm{~m}$ & 6.5 \\
Throttle constant & $c_{t h}$ & - & 1.7197 \\
Specific heat ratio of air & $\gamma$ & - & 1.4 \\
\hline
\end{tabular}




\section{Chapter 3}

\section{Controller Derivation}

\subsection{Integrator Backstepping}

Starting with the simple case of backstepping, as illustrated in [22], let us consider the system

$$
\dot{x}=f(x)+g(x) y, \quad f(0)=0,
$$

where $x \in \mathbf{R}^{\mathbf{n}}$ is the state and $y \in \mathbf{R}$ is the control input. The functions $f(x)$ and $g(x)$ are smooth in the domain $D \subset \mathbf{R}^{\mathbf{n}}$ that contains $x=0$ and $f(0)=0$. To design a state feedback controller that stabilizes the origin $(x=0)$, let us suppose that the system can be stabilized by the control law $y=\phi(x)$ with $\phi(0)=0$. Suppose further that we know a smooth positive definite Lyapunov function $V: \mathbf{R}^{\mathbf{n}} \rightarrow \mathbf{R}$ that satisfies the inequality

$$
\frac{\partial V}{\partial x}[f(x)+g(x) \phi(x)] \leq-W(x), \quad \forall x \in D,
$$

where $W: \mathbf{R}^{\mathbf{n}} \rightarrow \mathbf{R}$ is positive definite. Under this supposition, the control law $y=\phi(x)$ makes the origin $(x=0)$ of Equation 3.1 globally asymptotically stable. 
Now, adding an integrator to the system in Equation 3.1 results in the following system,

$$
\begin{aligned}
& \dot{x}=f(x)+g(x) y, \\
& \dot{y}=u .
\end{aligned}
$$

Let us suppose that the first component of the system in Equation 3.3 can be stabilized as discussed earlier with $y \in \mathbf{R}$ as the control input. If $W(x)$ is positive definite, then $V_{a}(x, y)=V(x)+\frac{1}{2}[y-\phi(x)]^{2}$ is the Lyapunov function for the system in Equation 3.3. It follows that there exist a control law $u=\phi_{a}(x, y)$ which makes the origin $(x=0, y=0)$ globally asymptotically stable. Such a control law is derived in [22] as,

$$
u=-\lambda_{1}[y-\phi(x)]+\frac{\partial \phi}{\partial x}[f(x)+g(x) y]-\frac{\partial V}{\partial x} g(x), \quad \lambda_{1}>0 .
$$

Recursive application of integrator backstepping can stabilize a system that has more than two states.

\subsection{Backstepping Design for Surge Control}

Integrator backstepping was applied in [4] for a two state compressor model. Here, the method is applied to a four state model which includes the piping dynamics. The 
full model is stated again here for convenience,

$$
\begin{aligned}
\dot{\Phi}_{c}= & B \omega_{H}\left(A_{1} \Phi_{c}^{3}+B_{1} \Phi_{c}^{2}+D_{1}+\frac{p_{o 1}}{\frac{1}{2} \rho_{o 1} U^{2}} k_{c l} \delta_{c l}-\Psi_{p}\right), \\
\dot{\Psi}_{p}= & \frac{\omega_{H}}{B}\left(\Phi_{c}-\Phi_{p}\right) \\
\dot{\Psi}_{t h}= & \frac{2 A_{12} A_{c}}{\rho_{u} U} \Phi_{p}+\frac{2 B_{12} A_{c}}{\rho_{u} U} u_{t h} c_{t h} \sqrt{\Psi_{t h}}, \\
\dot{\Phi}_{p}= & \frac{A_{21} \rho_{u} U}{2 A_{c}} \Psi_{t h}+A_{22} \Phi_{p}+\frac{B_{21} \rho_{u} U}{2 A_{c}} \Psi_{p}+\frac{\rho_{u} p_{o 1}}{\rho_{o 1} U A_{c}}\left(A_{21}+B_{21}\right)+\ldots \\
& +B_{22} u_{t h} c_{t h} \sqrt{\Psi_{t h}} .
\end{aligned}
$$

A change of variables can be applied to the model using Equation 3.6 in order to move the equilibrium points to the origin. The equilibrium values denoted by subscript eq show the intersection of the steady-state characteristics of the compressor characteristic and the throttle valve. The change of variables is given by,

$$
\begin{aligned}
& \xi_{1}=\Psi_{t h}-\Psi_{e q}, \\
& \xi_{2}=\Phi_{p}-\Phi_{e q}, \\
& \xi_{3}=\Psi_{p}-\Psi_{e q}, \\
& \xi_{4}=\Phi_{c}-\Phi_{e q} .
\end{aligned}
$$


Applying this change of variables, the model in Equation 3.5 becomes

$$
\begin{aligned}
\dot{\xi}_{1}= & \frac{2 A_{12} A_{c}}{\rho_{u} U}\left(\xi_{2}+\Phi_{e q}\right)+\frac{2 B_{12} A_{c}}{\rho_{u} U} u_{t h} c_{t h} \sqrt{\xi_{1}+\Psi_{e q}}-\frac{d \Psi_{e q}}{d t}, \\
\dot{\xi}_{2}= & \frac{A_{21} \rho_{u} U}{2 A_{c}}\left(\xi_{1}+\Psi_{e q}\right)+A_{22}\left(\xi_{2}+\Phi_{e q}\right)+\frac{B_{21} \rho_{u} U}{2 A_{c}}\left(\xi_{3}+\Psi_{e q}\right)+\ldots \\
& +\frac{\rho_{u} p_{o 1}}{\rho_{o 1} U A_{c}}\left(A_{21}+B_{21}\right)+B_{22} u_{t h} c_{t h} \sqrt{\xi_{1}-\Psi_{e q}}-\frac{d \Phi_{e q}}{d t} \\
\dot{\xi}_{3}= & \frac{\omega_{H}}{B}\left(\xi_{4}+\Phi_{e q}\right)-\frac{\omega_{H}}{B}\left(\xi_{2}+\Phi_{e q}\right)-\frac{d \Psi_{e q}}{d t}, \\
\dot{\xi}_{4}= & B \omega_{H}\left(A_{1}\left(\xi_{4}+\Phi_{e q}\right)^{3}+B_{1}\left(\xi_{4}+\Phi_{e q}\right)^{2}+D_{1}+\frac{p_{o 1}}{\frac{1}{2} \rho_{o 1} U^{2}} k_{c l} \delta_{c l}-\ldots\right. \\
& \left.-\xi_{3}-\Psi_{e q}\right)-\frac{d \Phi_{e q}}{d t}
\end{aligned}
$$

To make the expressions of the model in Equation 3.7 more simpler and convenient to use, the following algebraic manipulations are carried out,

$$
\dot{\xi}_{1}=f_{1}\left(\xi_{1}\right)+g_{1} \xi_{2}
$$

where $g_{1}$ and $f_{1}\left(\xi_{1}\right)$ are given as,

$$
\begin{aligned}
g_{1} & =\frac{2 A_{12} A_{c}}{\rho_{u} U} \\
f_{1}\left(\xi_{1}\right) & =\frac{2 A_{12} A_{c}}{\rho_{u} U}\left(\Phi_{e q}\right)+\frac{2 B_{12} A_{c}}{\rho_{u} U} u_{t h} c_{t h} \sqrt{\xi_{1}+\Psi_{e q}}-\frac{d \Psi_{e q}}{d t}, \quad f_{1}(0)=0 .
\end{aligned}
$$

Similarly,

$$
\dot{\xi}_{2}=f_{2}\left(\xi_{1}, \xi_{2}\right)+g_{2} \xi_{3}
$$


and $g_{2}$ and $f_{2}\left(\xi_{1}, \xi_{2}\right)$ are given as,

$$
\begin{aligned}
g_{2}= & \frac{B_{21} \rho_{u} U}{2 A_{c}} \\
f_{2}\left(\xi_{1}, \xi_{2}\right)= & \frac{A_{21} \rho_{u} U}{2 A_{c}}\left(\xi_{1}+\Psi_{e q}\right)+A_{22}\left(\xi_{2}+\Phi_{e q}\right)+\frac{B_{21} \rho_{u} U}{2 A_{c}}\left(\Psi_{e q}\right)+\ldots \\
& +\frac{\rho_{u} p_{o 1}}{\rho_{o 1} U A_{c}}\left(A_{21}+B_{21}\right)+B_{22} u_{t h} c_{t h} \sqrt{\xi_{1}-\Psi_{e q}}-\frac{d \Phi_{e q}}{d t}
\end{aligned}
$$

Same applies for the third system in the model,

$$
\dot{\xi}_{3}=f_{3}\left(\xi_{2}\right)+g_{3} \xi_{4},
$$

where $g_{3}$ and $f_{3}\left(\xi_{2}\right)$ are given as,

$$
\begin{aligned}
g_{3} & =\frac{\omega_{H}}{B} \\
f_{3}\left(\xi_{2}\right) & =\frac{\omega_{H}}{B}\left(\Phi_{e q}\right)-\frac{\omega_{H}}{B}\left(\xi_{2}+\Phi_{e q}\right)-\frac{d \Psi_{e q}}{d t} .
\end{aligned}
$$

Lastly,

$$
\dot{\xi}_{4}=f_{4}\left(\xi_{3}, \xi_{4}\right)+g_{4} \delta_{c l},
$$

where $g_{4}$ and $f_{4}\left(\xi_{3}, \xi_{4}\right)$ are given as,

$$
\begin{aligned}
g_{4}= & \frac{p_{o 1}}{\frac{1}{2} \rho_{o 1} U^{2}} B \omega_{H} k_{c l} \\
f_{4}\left(\xi_{3}, \xi_{4}\right)= & B \omega_{H}\left(A_{1}\left(\xi_{4}+\Phi_{e q}\right)^{3}+B_{1}\left(\xi_{4}+\Phi_{e q}\right)^{2}+D_{1}-\ldots\right. \\
& \left.-\xi_{3}-\Psi_{e q}\right)-\frac{d \Phi_{e q}}{d t} .
\end{aligned}
$$


The simplified model in Equation 3.5 can now be expressed in strict feedback from as,

$$
\begin{aligned}
& \dot{\xi}_{1}=f_{1}\left(\xi_{1}\right)+g_{1} \xi_{2}, \\
& \dot{\xi}_{2}=f_{2}\left(\xi_{1}, \xi_{2}\right)+g_{2} \xi_{3}, \\
& \dot{\xi}_{3}=f_{3}\left(\xi_{2}\right)+g_{3} \xi_{4}, \\
& \dot{\xi}_{4}=f_{4}\left(\xi_{3}, \xi_{4}\right)+g_{4} \delta_{c l},
\end{aligned}
$$

where $\delta_{c l}$ is the control law to be found that will globally asymptotically stabilize the entire compressor model. Now, for Equation 3.16a consider the Lyapunov candidate,

$$
V_{1}=\frac{1}{2} \xi_{1}^{2}
$$

Taking the derivative of $V_{1}$ with respect to time,

$$
\begin{aligned}
\dot{V}_{1} & =\frac{\partial V_{1}}{\partial \xi_{1}} \dot{\xi}_{1}, \\
& =\xi_{1}\left(f_{1}\left(\xi_{1}\right)+g_{1} \xi_{2}\right) .
\end{aligned}
$$

If the control law $\xi_{2}$ to the system in Equation 3.16a is given as,

$$
\begin{aligned}
\xi_{2} & =\alpha_{1}, \\
& =-\frac{1}{g_{1}}\left(f_{1}\left(\xi_{1}\right)+k_{1} \xi_{1}\right),
\end{aligned}
$$

where $k_{1}$ is positive, then,

$$
\begin{aligned}
\dot{V}_{1} & =-k_{1} \xi_{1}^{2}, \\
& <0
\end{aligned}
$$


results in $\left(\xi_{1}=0\right)$ for the system in Equation 3.16a being globally asymptotically stable.

Using input transformation for the system in Equation 3.16b, in which the control law $\xi_{3}$ is to be determined, we get

$$
\begin{aligned}
\alpha_{20} & =\xi_{3}, \\
& =\frac{1}{g_{2}}\left(\alpha_{2}-f_{2}\left(\xi_{1}, \xi_{2}\right)\right) .
\end{aligned}
$$

It is clear that the system in Equation $3.16 \mathrm{~b}$ can now be written as

$$
\dot{\xi}_{2}=\alpha_{2}
$$

Now, applying the recursive step to integrator backstepping,

$$
V_{2}=V_{1}+\frac{1}{2}\left(\xi_{2}-\alpha_{1}\right)^{2}
$$

will be the Lyapunov candidate that makes Equation 3.16a and Equation 3.16b globally asymptotically stable. Then,

$$
\begin{aligned}
\dot{V}_{2} & =\frac{\partial V_{1}}{\partial \xi_{1}} \dot{\xi}_{1}+\left(\xi_{2}-\alpha_{1}\right)\left(\dot{\xi}_{2}-\frac{\partial \alpha_{1}}{\partial \xi_{1}} \dot{\xi}\right), \\
& =\frac{\partial V_{1}}{\partial \xi_{1}}\left(f_{1}\left(\xi_{1}\right)+g_{1} \xi_{2}\right)+\left(\xi_{2}-\alpha_{1}\right)\left(\dot{\xi}_{2}-\frac{\partial \alpha_{1}}{\partial \xi_{1}} \dot{\xi}_{1}\right), \\
& =\underbrace{\frac{\partial V_{1}}{\partial \xi_{1}}\left(f_{1}\left(\xi_{1}\right)+g_{1} \alpha_{2}\right)}_{<0}+\frac{\partial V_{1}}{\partial \xi_{1}}\left(g_{1} \xi_{2}-g_{1} \alpha_{1}\right)+\left(\xi_{2}-\alpha_{1}\right)\left(\alpha_{2}-\frac{\partial \alpha_{1}}{\partial \xi_{1}}\left(f_{1}\left(\xi_{1}\right)+g_{1} \xi_{2}\right)\right) .
\end{aligned}
$$

Choosing the control law $\alpha_{2}$ as follows,

$$
\left.\alpha_{2}=\frac{\partial \alpha_{1}}{\partial \xi_{1}}\left(f_{1}\left(\xi_{1}\right)+g_{1} \xi_{2}\right)\right)-\frac{\partial V_{1}}{\partial \xi_{1}} g_{1}-k_{2}\left(\xi_{2}-\alpha_{1}\right)
$$


where $k_{2}$ is positive results in

$$
\begin{aligned}
\dot{V}_{2} & =\dot{V}_{1}-\frac{1}{2} k_{2}\left(\xi_{2}-\alpha_{1}\right)^{2}, \\
& <0,
\end{aligned}
$$

making $\left(\xi_{1}=0, \xi_{2}=0\right)$ for the systems in Equation 3.16a and Equation 3.16b globally asymptotically stable. Using an input transformation once again for the system in Equation 3.16c where the control law $\xi_{4}$ is to be determined,

$$
\begin{aligned}
\alpha_{30} & =\xi_{4}, \\
& =\frac{1}{g_{3}}\left(\alpha_{3}-f_{3}\left(\xi_{2}\right)\right),
\end{aligned}
$$

will result in,

$$
\dot{\xi}_{3}=\alpha_{3}
$$

Representing the systems in Equation 3.16a and Equation 3.16b as one vector system as shown in Equation 3.29a,

$$
\begin{aligned}
& \dot{\boldsymbol{\Gamma}}=\mathbf{f}_{\boldsymbol{\Gamma}}+\mathbf{g}_{\boldsymbol{\Gamma}} \xi_{\mathbf{3}}, \\
& \dot{\xi}_{3}=\alpha_{3},
\end{aligned}
$$

where

$$
\dot{\boldsymbol{\Gamma}}=\left[\begin{array}{c}
\dot{\xi}_{1} \\
\dot{\xi}_{2}
\end{array}\right], \mathbf{f}_{\boldsymbol{\Gamma}}=\left[\begin{array}{c}
f_{1}\left(\xi_{1}\right)+g_{1} \xi_{2} \\
f_{2}\left(\xi_{1}, \xi_{2}\right)
\end{array}\right], \mathbf{g}_{\boldsymbol{\Gamma}}=\left[\begin{array}{c}
0 \\
g_{2}
\end{array}\right]
$$


To find a globally asymptotically stabilizing controller $\alpha_{30}$ for system in Equation 3.29, let us consider the Lyapunov candidate,

$$
\begin{aligned}
V_{3}= & V_{2}+\frac{1}{2}\left(\xi_{3}-\alpha_{20}\right)^{2}, \\
\dot{V}_{3}= & \frac{\partial V_{2}}{\partial \xi_{1}} \dot{\xi}_{1}+\frac{\partial V_{2}}{\partial \xi_{2}} \dot{\xi}_{2}+\left(\xi_{3}-\alpha_{20}\right)\left(\dot{\xi}_{3}-\frac{\partial \alpha_{20}}{\partial \xi_{1}} \dot{\xi}_{1}-\frac{\partial \alpha_{20}}{\partial \xi_{2}} \dot{\xi}_{2}\right), \\
= & \frac{\partial V_{2}}{\partial \xi_{1}} \dot{\xi}_{1}+\frac{\partial V_{2}}{\partial \xi_{2}}\left(f_{2}\left(\xi_{1}, \xi_{2}\right)+g_{2} \xi_{3}-g_{2} \alpha_{20}+g_{2} \alpha_{20}\right)+\ldots \\
& \left(\xi_{3}-\alpha_{20}\right)\left(\alpha_{3}-\frac{\partial \alpha_{20}}{\partial \xi_{1}} \dot{\xi}_{1}-\frac{\partial \alpha_{20}}{\partial \xi_{2}} \dot{\xi}_{2}\right), \\
= & \underbrace{\frac{\partial V_{2}}{\partial \xi_{1}} \dot{\xi}_{1}-\frac{\partial \alpha_{1}}{\partial \xi_{1}}\left(\xi_{2}-\alpha_{1}\right) \dot{\xi}_{1}+\left(\xi_{2}-\alpha_{1}\right)\left(f_{2}\left(\xi_{1}, \xi_{2}\right)+g_{2} \alpha_{20}\right)+\ldots}_{<0 \text { if } \alpha_{20} \text { from Equation } 3.21 \text { is substituted }}+ \\
& \left(\xi_{3}-\alpha_{20}\right)\left(\alpha_{3}-\frac{\partial \alpha_{20}}{\partial \xi_{1}} \dot{\xi}_{1}-\frac{\partial \alpha_{20}}{\partial \xi_{2}} \dot{\xi}_{2}+\frac{\partial V_{2}}{\partial \xi_{2}} g_{2}\right) .
\end{aligned}
$$

We can now choose the control law $\alpha_{3}$ as,

$$
\alpha_{3}=\frac{\partial \alpha_{20}}{\partial \xi_{1}} \dot{\xi}_{1}+\frac{\partial \alpha_{20}}{\partial \xi_{2}} \dot{\xi}_{2}-\frac{\partial V_{2}}{\partial \xi_{2}} g_{2}-k_{3}\left(\xi_{3}-\alpha_{20}\right),
$$

and for $k_{3}$ positive we get,

$$
\begin{aligned}
\dot{V}_{3} & =\dot{V}_{2}-\frac{1}{2} k_{3}\left(\xi_{3}-\alpha_{20}\right)^{2} \\
& <0
\end{aligned}
$$

clearly making $\left(\xi_{1}=0, \xi_{2}=0, \xi_{3}=0\right)$ for the system in Equation 3.29 globally asymptotically stable.

For the last backstepping step to find the control law $\delta_{c l}$ to stabilize the entire system in Equation 3.16, let us combine the systems in Equation 3.16a, Equation 
3.16b and Equation 3.16c into one vector system as in Equation 3.33a,

$$
\begin{aligned}
& \dot{\boldsymbol{\Upsilon}}=\mathbf{f}_{\Upsilon}+\mathbf{g}_{\Upsilon} \xi_{\mathbf{4}}, \\
& \dot{\xi}_{4}=\alpha_{4},
\end{aligned}
$$

where

$$
\dot{\boldsymbol{\Upsilon}}=\left[\begin{array}{c}
\dot{\xi}_{1} \\
\dot{\xi}_{2} \\
\dot{\xi}_{3}
\end{array}\right], \mathbf{f}_{\Upsilon}=\left[\begin{array}{c}
f_{1}\left(\xi_{1}\right)+g_{1} \xi_{2} \\
f_{2}\left(\xi_{1}, \xi_{2}\right)+g_{2} \xi_{3} \\
f_{3}\left(\xi_{2}\right)
\end{array}\right], \mathbf{g} \Upsilon_{\Upsilon}=\left[\begin{array}{c}
0 \\
0 \\
g_{3}
\end{array}\right] .
$$

Making the final input transformation

$$
\begin{aligned}
\alpha_{40} & =\delta_{c l}, \\
& =\frac{1}{g_{4}}\left(\alpha_{4}-f_{4}\left(\xi_{3}, \xi_{4}\right)\right),
\end{aligned}
$$

which makes

$$
\dot{\xi}_{4}=\alpha_{4},
$$


we can find a globally asymptotically stabilizing controller $\delta_{c l}$ for the system in Equation 3.33a. Let us consider the Lyapunov candidate

$$
\begin{aligned}
V_{4}= & V_{3}+\frac{1}{2}\left(\xi_{4}-\alpha_{30}\right)^{2}, \\
\dot{V}_{4}= & \left.\frac{\partial V_{3}}{\partial \xi_{1}} \dot{\xi}_{1}+\frac{\partial V_{3}}{\partial \xi_{2}} \dot{\xi}_{2}+\frac{\partial V_{3}}{\partial \xi_{3}} \dot{\xi}_{3}+\left(\xi_{4}-\alpha_{30}\right)\left(\dot{\xi}_{4}-\frac{\partial \alpha_{30}}{\partial \xi_{1}} \dot{\xi}_{1}-\frac{\partial \alpha_{30}}{\partial \xi_{2}} \dot{\xi}_{2}\right)-\frac{\partial \alpha_{30}}{\partial \xi_{3}} \dot{\xi}_{3}\right), \\
= & \frac{\partial V_{1}}{\partial \xi_{1}} \dot{\xi}_{1}-\frac{\partial \alpha_{1}}{\partial \xi_{1}}\left(\xi_{2}-\alpha_{1}\right)-\frac{\partial \alpha_{20}}{\partial \xi_{1}} \dot{\xi}_{1}\left(\xi_{3}-\alpha_{20}\right)+\xi_{2} \dot{\xi}_{2}-\frac{\partial \alpha_{20}}{\partial \xi_{2}} \dot{\xi}_{2}+\ldots \\
& \left.\left.\frac{\partial V_{3}}{\partial \xi_{3}}\left(f_{3}\left(\xi_{2}\right)+g_{3} \alpha_{30}-g_{3} \alpha_{30}\right)+g_{3} \xi_{4}\right)+\left(\xi_{4}-\alpha_{30}\right)\left(\dot{\xi}_{4}-\frac{\partial \alpha_{30}}{\partial \xi_{1}} \dot{\xi}_{1}-\frac{\partial \alpha_{30}}{\partial \xi_{2}} \dot{\xi}_{2}\right)-\frac{\partial \alpha_{30}}{\partial \xi_{3}} \dot{\xi}_{3}\right), \\
= & \underbrace{\frac{\partial V_{1}}{\partial \xi_{1}}\left(f_{1}\left(\xi_{1}\right)+g_{1} \alpha_{1}\right)+\left(\xi_{2}-\alpha_{1}\right)\left(\frac{\partial V_{1}}{\partial \xi_{1}} g_{1}-\frac{\partial \alpha_{1}}{\partial \xi_{1}} \dot{\xi}_{1}-\frac{\partial \alpha_{20}}{\partial \xi_{1}} \dot{\xi}_{1}\left(\xi_{3}-\alpha_{20}\right) \xi_{2} \dot{\xi}_{2} \ldots\right.}{ } \underbrace{\frac{\partial \alpha_{20}}{\partial \xi_{2}} \dot{\xi}_{2}+\xi_{3}\left(f_{3}\left(\xi_{2}\right)+g_{3} \alpha_{30}\right)}_{<0 \text { if } \alpha_{30} \text { from Equation } 3.27 \text { is substituted }}+\left(\xi_{4}-\alpha_{30}\right)\left(\dot{\xi}_{4}-\frac{\partial \alpha_{30}}{\partial \xi_{1}} \dot{\xi}_{1}-\frac{\partial \alpha_{30}}{\partial \xi_{2}} \dot{\xi}_{2}\right)-\frac{\partial \alpha_{30}}{\partial \xi_{3}} \dot{\xi}_{3}) .
\end{aligned}
$$

Now, choosing the control law $\alpha_{4}$ as

$$
\alpha_{4}=\frac{\partial \alpha_{30}}{\partial \xi_{1}} \dot{\xi}_{1}+\frac{\partial \alpha_{30}}{\partial \xi_{2}} \dot{\xi}_{2}+\frac{\partial \alpha_{30}}{\partial \xi_{3}} \dot{\xi}_{3}-\frac{\partial V_{3}}{\partial \xi_{3}} g_{3}-k_{4}\left(\xi_{4}-\alpha_{30}\right)
$$

where $k_{4}$ is positive will result in

$$
\begin{aligned}
\dot{V}_{4} & =\dot{V}_{3}-\frac{1}{2} k_{4}\left(\xi_{4}-\alpha_{30}\right)^{2}, \\
& <0
\end{aligned}
$$

which makes $\left(\xi_{1}=0, \xi_{2}=0, \xi_{3}=0, \xi_{4}=0\right)$ in Equation 3.33 globally asymptotically stable. The control law that will stabilize the entire compressor model in Equation 3.5 can finally be derived as

$$
\delta_{c l}=\frac{1}{g_{4}}\left(\frac{\partial \alpha_{30}}{\partial \xi_{1}} \dot{\xi}_{1}+\frac{\partial \alpha_{30}}{\partial \xi_{2}} \dot{\xi}_{2}+\frac{\partial \alpha_{30}}{\partial \xi_{3}} \dot{\xi}_{3}-\frac{\partial V_{3}}{\partial \xi_{3}} g_{3}-k_{4}\left(\xi_{4}-\alpha_{30}\right)-f_{4}\left(\xi_{3}, \xi_{4}\right)\right) .
$$




\subsection{Simulation Results}

The model in Equation 3.5 was simulated in MATLAB and Simulink both with and without the controller engaged. The behavior of the model was analyzed for both the linear and nonlinear tip clearance effects. The simulation results presented are for the nonlinear effects of the impeller tip clearance. The linearized model was only used for controller derivation.

The simulations demonstrated that surge begins when the throttle valve opening is at $18.1 \%$ opening. That observed, in one of the simulations, the throttle valve was varied from $20 \%$ down to $16.5 \%$ and then up again to $20 \%$ opening in a period of 20 seconds, it then stayed at $20 \%$ for another 10 seconds. In the second simulation the throttle valve was closed from $20 \%$ to $16.5 \%$ in 10 seconds and then stayed at $16.5 \%$ for a further 20 seconds. It was observed that decreasing the rate at which the throttle valve opening changes, improved the controller performance.

For the first simulation in Figure 3.1, the plot in (a) shows the throttle valve closing to $16.5 \%$ and then opening again. The plot in (b) demonstrates the surge limit cycle. It becomes a limit cycle because as the oscillations happen, the plenum is emptied and filled again with air hence the fluctuations in pressure rise $\Psi_{p}$ shown in (c). Similar fluctuations are also observed in the mass flow rate $\Phi_{c}$. Plot $(d)$ is always at zero because the controller is not engaged. These surge plots do not differ significantly for both linear and nonlinear impeller tip clearance effects.

When the surge controller is engaged, the rapid fluctuations that were observed in the pressure rise $\Psi_{p}$ are completely stabilized. Figure 3.2 plot (a) shows the throttle valve closing to $16.5 \%$ and then opening again back to $20 \%$. The surge limit cycle is eliminated in (b) and the fluctuation observed in Figure 3.1 have been stabilized. The plot in (c) clearly illustrates that as the compressor goes deep into surge, the pressure rise $\Psi_{p}$ begins to drop. Operating a compressor deep into surge is not necessary as it requires more control effort yet not yielding higher pressure rises. The best operating 

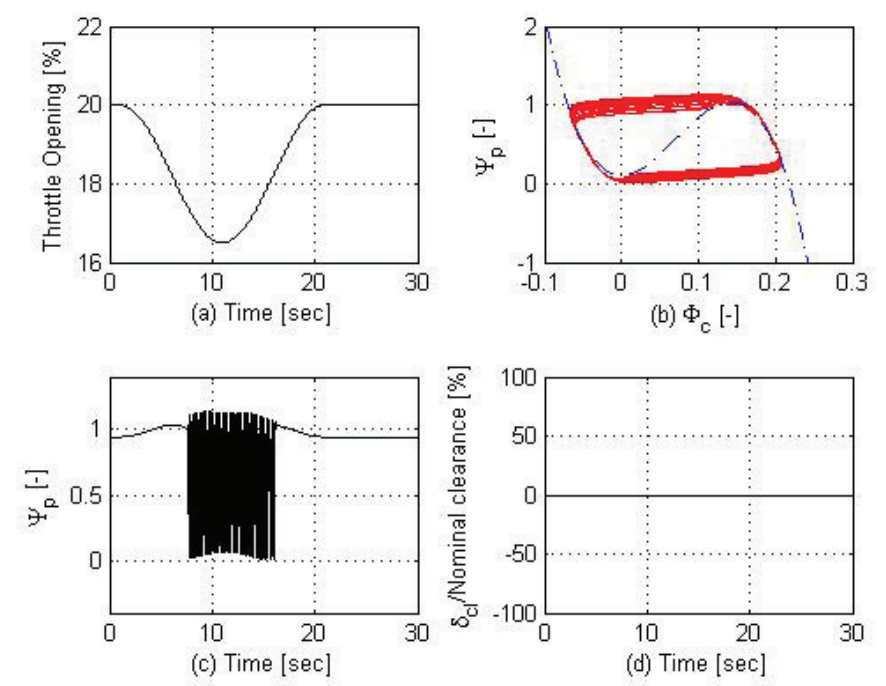

Figure 3.1: Demonstration of a surge interval with controller turned off
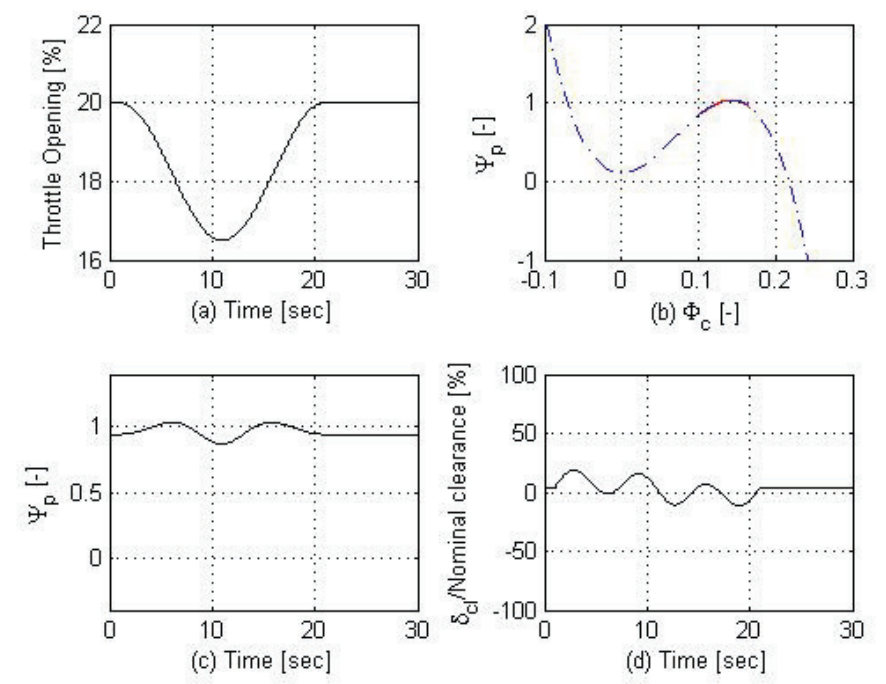

Figure 3.2: Controller engaged and surge interval stabilized

point would be at the surge limit line, where the maximum possible pressure rises can be harvested without the need to worry about surge. The controller is shown in plot (d).

The second simulation in Figure 3.3 shows the throttle valve closing from $20 \%$ to $16.5 \%$ and staying there throughout. The successful control of continued surge is shown in Figure 3.4. 

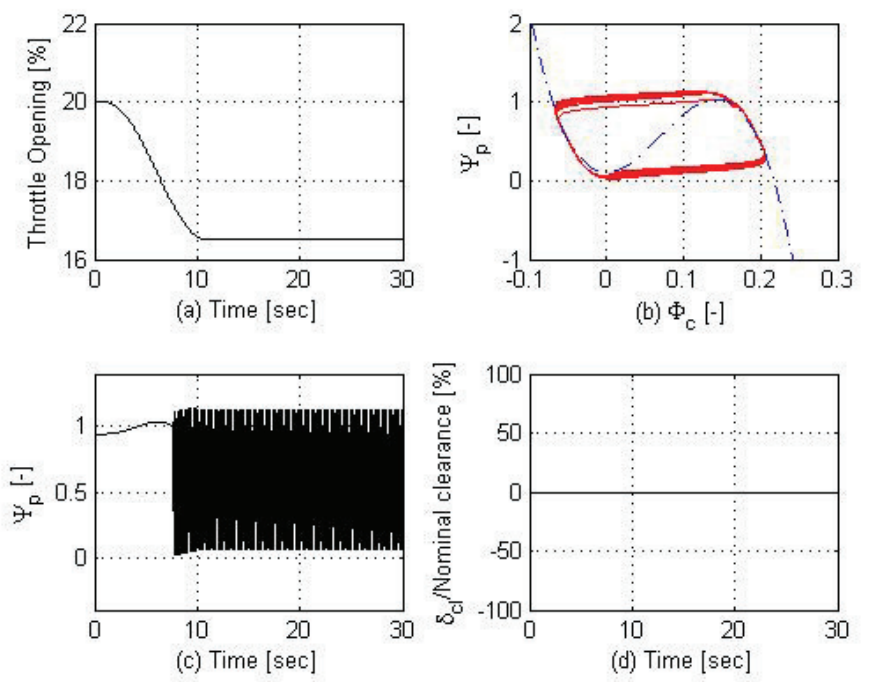

Figure 3.3: Demonstration of continued surge
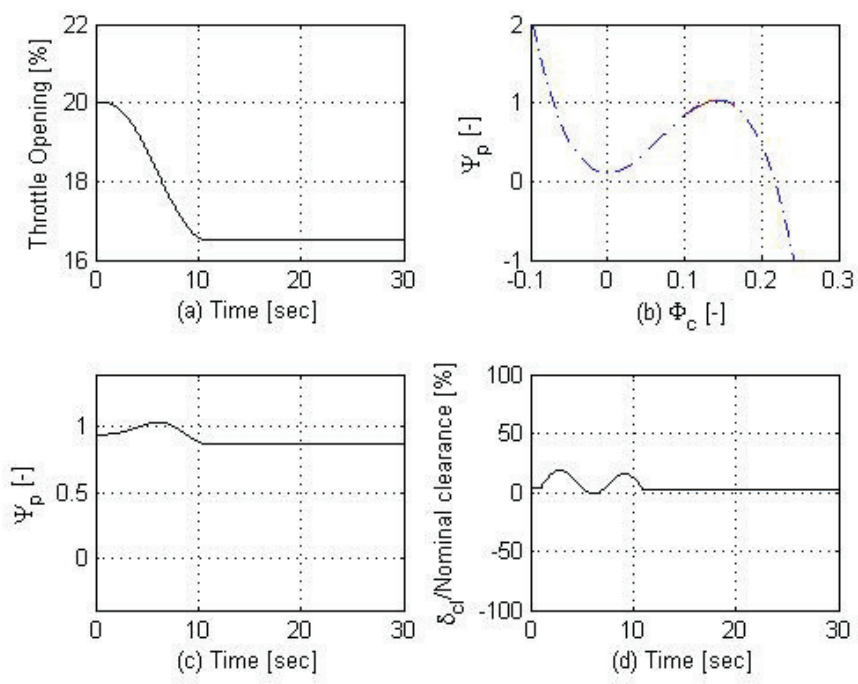

Figure 3.4: An engaged controller stabilizes surge

A closer look at what happens to the operation of the compressor on the characteristic curve is captured in Figure 3.5. The $20 \%$ arrow points at the operating point when the throttle valve is at $20 \%$. This operating point is in the stable region. As the throttle valve is closed beyond $18.1 \%$, it enters the unstable region. The $16.5 \%$ arrow points at the operating point when the throttle valve is at $16.5 \%$ and the operating point deep into surge. Once again, it is clear that the best operating point would be 


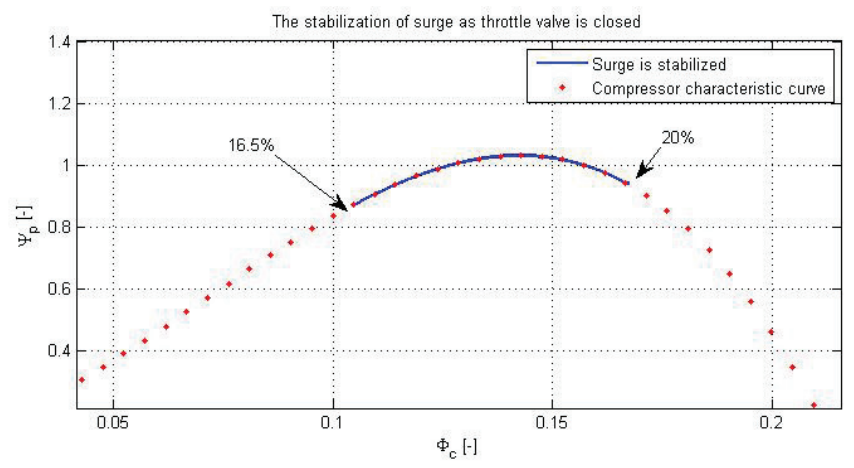

Figure 3.5: Demonstration of surge control on the compressor characteristic curve somewhere around $18.1 \%$ throttle valve opening. The backstepping controller derived was able to stabilize surge and peaked at $19 \%$ of the nominal impeller tip clearance. The throttle valve opening could be closed as low as $14.7 \%$ and still stabilization was achieved. Closing the throttle valve below $14.7 \%$ resulted in the physical constraint on the available impeller clearance being violated. 


\section{Chapter 4}

\section{Conclusions and Future Work}

\subsection{Conclusions}

The design and simulation of an active surge controller was discussed in this thesis. The surge controller was designed for a centrifugal compressor that employed radial AMBs for rotor levitation and an axial AMB for modulation of the impeller tip clearance. The ability to modulate the impeller tip clearance was utilized to stabilize surge in compression systems. The backstepping control method was applied to the compressor model to derive a control law that stabilized surge.

Chapter 1 presented the basic concepts on types of compressors and how they operate. A centrifugal compressor was used for work related to this thesis. This chapter introduced surge and discussed ways to control it. The chapter also discussed the previous work done on the centrifugal compressor.

Chapter 2 presented how the Greitzer model was used to develop a model for the centrifugal compressor that was used in this thesis. The effects of impeller tip clearance were modeled and linearized in this chapter. The inclusion of the piping model and how it was experimentally validated was also discussed. The chapter concluded with the assembling of the overall model. 
The controller derivation was presented in Chapter 3. An introduction to integrator backstepping was first introduced in Chapter 3 before it was applied recursively to stabilize the full assembled compressor model. The simulations showed that the control law derived was able to stabilize surge. The throttle valve opening was closed from $20 \%$ to $16.5 \%$ passing through the surge point at $18.1 \%$ in the process. The controller peaked at $19 \%$ of nominal impeller tip clearance. The simulation results looked very promising.

Finally, this chapter, Chapter 4, provides a closing conclusion and discusses the potential experimental implementation of the surge controller derived. A discussion on how the work presented in this thesis can be advanced in the future is also presented. Lastly, from a business perspective, this chapter looks at the economic viability of active surge control for compressors using AMBs and what the future holds for the technology.

\subsection{Future Experimental Work}

The simulation results demonstrated the potential of the backstepping control law in the stabilization of surge. However, for practical implementation, the effects of AMBs dynamics would need to be incorporated into the controller derivation. An attempt to include the AMBs dynamics as a low pass Butterworth filter of order three with a cutoff frequency of $75 \mathrm{~Hz}$ showed unstable behavior. Once the control law can be shown to work with AMBs dynamics included, the experimental implementation of the controller can involve porting the MATLAB and Simulink programs that run the controller into the $\mathrm{C}$ programming language . Once the $\mathrm{C}$ code is generated, it can then be run on the control computer.

The backstepping controller has the theoretical ability to stabilize surge on the entire compressor characteristic curve when there are disturbances downstream the 
compressor. An introduction of disturbances in the throttle valve opening should be practically employed to evaluate the robustness of the controller. As this experimental work is carried out, attention should be paid to the physical constraint on the available impeller tip clearance. The control effort required as the compressor goes deep into surge is limited by the nominal impeller tip clearance.

\subsection{Future Research Work}

The backstepping controller that was derived in this thesis has the capability of stabilizing surge using the knowledge of the throttle valve opening and the compressor characteristic curve. Whilst the knowledge of the throttle valve opening is necessary for correct controller operation, full knowledge of the compressor characteristic curve might be done away with. Adaptive parameter estimation methods can be explored in cases where the form of the characteristic curve is known but the coefficients are not. This is one area of potential future research work.

Another area of future research is the adaptive engagement of the surge controller. When there is no surge, the controller should be off and only to engage when surge starts to initiate. This automatic turning on and off of the controller can be made possible through monitoring changes in pressure rises and mass flow rates in the compressor.

\subsection{Economic Viability of Active Magnetic Bear- ings}

Over the past years, the costs of incorporating magnetic bearings in rotating machinery have been exorbitant. However, through standardization, integration and manufacturing advances these costs have been declining [23]. Despite the still high initial 
investment costs for AMBs, there are numerous benefits that make the technology economically viable.

According to SKF, a magnetic bearings manufacturer, AMBs provide the highest energy efficiency in comparison to other types of drives when used in electric motors. When used in compressors and other rotating machinery, efficiency is improved because the mechanical bearings related energy losses are completely eliminated. In addition, the absence of mechanical bearings also improves the service life because there will be no component wear. This guarantees an increase in production. Another benefit associated with the absence of mechanical bearings is the reduced long term cost of maintenance and spare parts. The final benefit in this regard is the absence of minimum speed requirements. The only concern will be designing the rotor for critical speeds and not incorporating bearings in the design process.

The other benefit that AMBs provide is increased reliability. The mean time between failure of the electronics involved in AMBs is very high and this ensures that machinery is highly reliable and has increased availability. Because of the electronics involved, advanced condition monitoring is made easier and the monitoring and control of operation can be achieved remotely. The monitoring and control ensures a significantly low possibility of damage to the machinery as a result of surge and other undesirable operations.

In this age where most engineering solutions are required to meet strict environmental constraints, solutions that use AMBs are well posed for success. Since there are no mechanical parts that rub against each other, AMBs are oil-free. This makes them contamination-free. Machinery that employs AMBs can handle sub-sea operation where harsh pressure conditions present a challenge for machinery that requires oil in their mechanical bearings. The minimized housing vibrations associated with AMBs also eliminate the concern for excessive noise pollution and reduce the costs of investing in machine foundations. 
As the manufacturing of AMBs continue to advance, a reduction in costs is expected. This reduction in costs coupled with the numerous benefits discussed in this section pave way for a bright future in AMB solutions. 


\section{Bibliography}

[1] Se Young Yoon. Surge Control of Active Magnetic Bearing Suspended Centrifugal Compressors. PhD thesis, Department of Electrical and Computer Engineering, University of Virginia, 2012.

[2] G. K. McMillan. Centrifugal and Axial Compressor Control. Instrument Society of America, USA, 1983.

[3] R. S. Brown. Compressors, Selection and Sizing. Elsevier Inc, 2005.

[4] Dorsa Sanadgol. Active control of surge in centrifugal compressor. PhD thesis, Department of Mechanical and Aerospace Engineering, University of Virginia, 2006.

[5] P. C Hanlon. Compressor Handbook. McGraw-Hill, 2001.

[6] K. H. Ludtke. Process Centrifugal Compressors: Basics, Function, Operation, Design. Springer, 2004.

[7] S. Y. Yoon, Z. Lin, and P. E. Allaire. Control of Surge in Centrifugal Compressors by Active Magnetic Bearings, Theory and Implementation. Springer, 2013.

[8] API 617. Axial and centrifugal compressors and expander-compressors for petroleum, chemicals and gas industry services. American Petroleum Institute, Washington, D.C., seventh edition, July 2002.

[9] Kin Tien Lim. Commissioning and characterization of a centrifugal compressor surge test rig. Master's thesis, Department of Mechanical and Aerospace Engineering, University of Virginia, 2006.

[10] A. H. Epstein, E. F. Williams, and E. M. Greitzer. Active suppression of aerodynamic instabilities in turbomachinery. Journal of Propulsion and Power, 5, March 1989.

[11] C. Rodgers. Centrifugal compressor inlet guide vanes for increased surge margin. Journal of Turbomachinery, 125, October 2003.

[12] G. J. Skoch. Experimental investigation of centrifugal compressor stabilization techniques. Journal of Turbomachinery, 125, October 2003. 
[13] M. Krstic, D. Fontaine, P. V. Kokotovic, and J. D. Paduano. Useful nonlinearities and global stabilization of bifurcations in a model of jet enginer surge and stall. IEEE Transactions on Automatic Control, 43, December 1998.

[14] G. Schweitzer and E. H. Maslen. Magnetic Bearings. Springer, 2013.

[15] N. Brown. High-speed compressor facility electromechanical design. Master's thesis, University of Virginia, 2005.

[16] E. M. Greitzer. Surge and rotating stall in axial flow compressors, part i, ii. ASME Journal of Engineering for Power, 120, 1976.

[17] F. P. T. Willems. Modeling and Bounded Feedback Stabilization of Centrifugal Compressor Surge. PhD thesis, Technische Universiteit Eindhoven, 2000.

[18] M. B. Graf, T. S. Wong, E. M. Greitzer, F. E. Marble, C. S. Tan, H. W. Shin, and D. C. Wisler. Effects of nonaxisymmetric tip clearance on axial compressor performance and stability. ASME Journal of Turbomachinery, 120, October 1998.

[19] R. C. Pampreen. Small turbomachinery compressor and fan aerodynamics. ASME Journal of Engineering for Power, 95, 1973.

[20] N. A. Cumpsty. Compressor Aerodynamics. Longman Scientific Technical, 1989.

[21] W. C. Yang and W. E. Tobler. Dissipative modal approximation of fluid transmission lines using linear friction model. ASME Journal of Dynamic Systems, Measurements, and Control, 113, 1991.

[22] H. K. Khalil. Nonlinear Systems. Prentice Hall, 2002.

[23] V. Iannello. Advances in magnetic bearings, 2010. Available at www.synchrony.com/documents/Advances in Magnetic Bearings.pdf. 